\title{
Argile et bouse de vache. Les récipients de la région Jbâla (Maroc)
}

Juan José Ibáñez, Leonor Peña-Chocarro, Lydia Zapata, Jesús Emilio González Urquijo et Marta Moreno García

\section{(2) OpenEdition}

\section{Journals}

Édition électronique

URL : https://journals.openedition.org/tc/240

DOI : $10.4000 /$ tc. 240

ISSN : 1952-420X

Éditeur

Éditions de l'EHESS

\section{Édition imprimée}

Date de publication : 1 mars 2002

ISSN : 0248-6016

\section{Référence électronique}

Juan José Ibáñez, Leonor Peña-Chocarro, Lydia Zapata, Jesús Emilio González Urquijo et Marta Moreno García, «Argile et bouse de vache. Les récipients de la région Jbâla (Maroc) », Techniques \& Culture [En ligne], 38 | 2002, mis en ligne le 11 juillet 2006, consulté le 29 septembre 2022. URL : http:// journals.openedition.org/tc/240 ; DOI : https://doi.org/10.4000/tc.240

Ce document a été généré automatiquement le 29 septembre 2022.

Tous droits réservés 


\title{
Argile et bouse de vache. Les récipients de la région Jbâla (Maroc)
}

\author{
Juan José Ibáñez, Leonor Peña-Chocarro, Lydia Zapata, Jesús Emilio \\ González Urquijo et Marta Moreno García
}

La région de Jbâla, dans le nord-ouest du Maroc (voir carte), présente un très grand intérêt anthropologique en raison de la préservation de formes de vie traditionnelles (Vignet-Zunz 1993). Depuis 1997, nous y développons un projet de recherche ethnoarchéologique qui repose sur l'étude de diverses techniques d'artisanats traditionnels ${ }^{1}$.

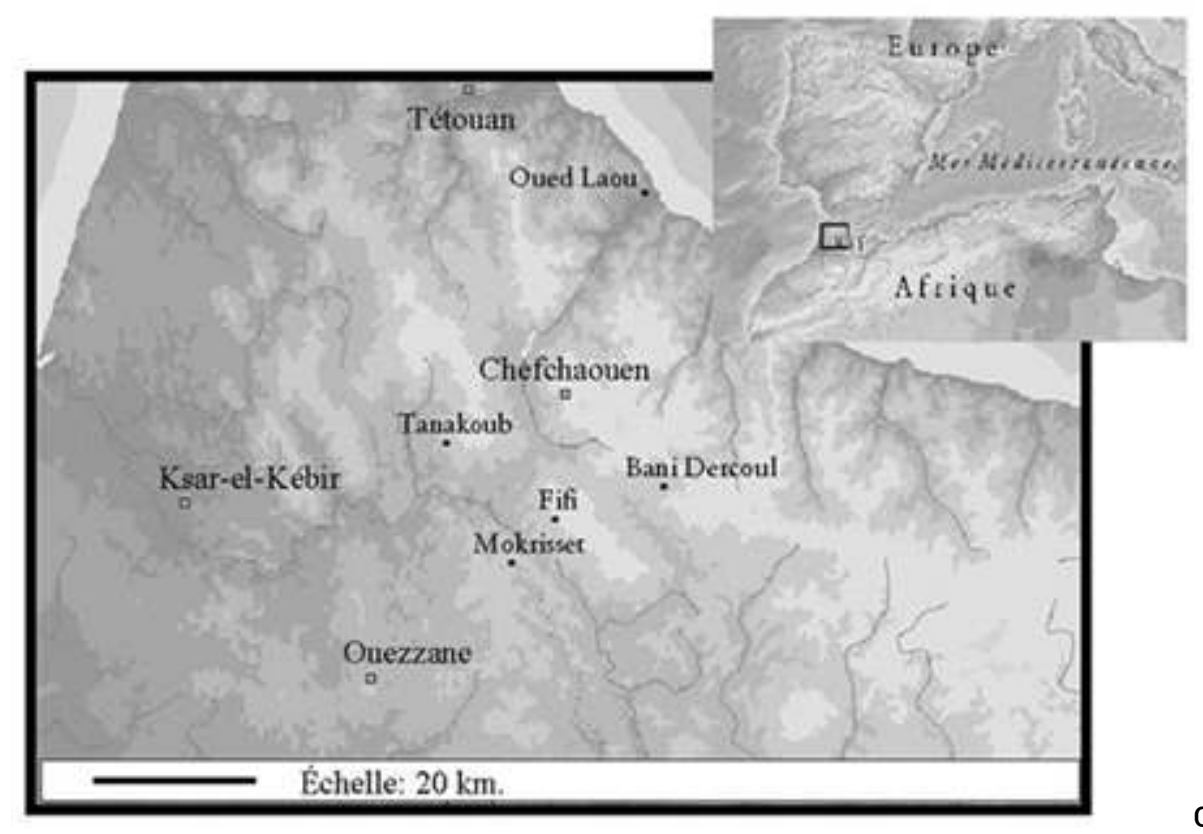

de la région

2 Dans cet article, nous aborderons l'un des aspects les plus remarquables du riche patrimoine technique traditionnel de la région, la fabrication et l'utilisation de récipients en argile non cuite et en bouse de vache. Puis nous présenterons leur 
processus de fabrication; enfin nous attirerons l'attention des archéologues sur la possibilité de repérer des objets semblables dans certains sites étudiés.

Les récipients

Les récipients de la région Jbâla, destinés à différents types de contenus, sont fabriqués avec trois matériaux différents : bouse de vache, parfois couverte d'une couche d'argile; argile; argile mélangée à de la bouse de vache.

Deux catégories morpho-fonctionnelles peuvent être distinguées : des contenants de forme cylindrique et souvent légèrement fuselée, d'une hauteur variant de $80 \mathrm{~cm}$ à plus de $2 \mathrm{~m}$, pour le stockage des produits agricoles secs; d'autres, plus petits, plus ouverts, de type bol, utilisés pour la nourriture donnée aux animaux et pour apporter les céréales au moulin rotatif.

5 En croisant la variable matière première avec la morphologie de l'objet, ont été répertoriés quatre types de récipients : ceux de morphologie cylindrique, élaborés 1) en argile non cuite, 2) en bouse de vache, 3) en argile mélangée avec la bouse, et 4) les bols, exclusivement fabriqués en bouse de vache.

6 En ce qui concerne les récipients cylindriques de stockage, nous en avons trouvé en argile non cuite dans divers douar (petits villages) situés entre le fleuve Ouerga et la chaîne montagneuse rifaine, au sud et à l'ouest de la ville de Ouezzane (Douar de Kidiua Hainuna, Ksera et Slit), ainsi qu'à Oued Lau (Douar de Ifran Ali ), sur la côte nord. La variante en bouse de vache a été repérée au sud de Chefchaouen, autour des villages de Mokrisset (Douar de Briet, Kalaa, Saara et Uled Shkirah), Tanakoub (Douar de Harrakah, Hommar et Harrakin) et Fifi (Douar de Teiyut). Celle faite d'un mélange de bouse et d'argile a été observée dans quelques douar entre Fifi et Mokrisset (Douar de Der Hok), et dans la région de Bani Dercoul (Douar de Tassaft). Ces récipients cylindriques, quelle qu'en soit la matière première, sont parfois appelés tabtoba, parfois tonna, selon les villages. Les bols en bouse de vache ont été repérés dans les douar proches de Tanakoub (Douar de Hommar et Harrakah), ou ils sont nommés elgelah ou tabtoba.

7 La distribution géographique de ces quatre types de récipients correspond aux territoires des tribus Bani Mesgilda, Bani Mestara, Ghzawa, El-Akhmas et Bani Saïd. Une certaine cohérence semble exister en fonction de la matière première. Les objets fabriqués en bouse de vache, seule ou mêlée d'argile, se répartissent dans la frange centrale montagneuse (Mokrisset, Fifi, Tanakoub, Bani Dercoul), alors que ceux en argile non cuite sont vraisemblablement propres au sud de la chaîne (Ouezzan) et à la zone côtière du nord (Oued Laou).

8 Cette distribution est liée, nous semble-t-il, aux données climatiques, comme le suggère l'analyse pluviométrique. Sous l'influence des masses originaire de l'Atlantique, les versants nord-ouest du Rif sont plus humides; les précipitations, plus abondantes en altitude, dépassent les $1500 \mathrm{~mm}$ annuels sur les sommets, alors que sur la côte et vers le sud, elles atteignent à peine $500 \mathrm{~mm}$ (Chikhi \& El Abdellaoui $1996: 257$, carte 2). Il en résulte une disponibilité différentielle de la matière première : les régions humides et montagneuses étant plus aptes à l'élevage, le nombre de vaches y est plus important que dans les régions plus sèches du nord et du sud de la chaîne montagneuse. Un deuxième facteur explicatif est peut être la meilleure capacité isolante de la bouse au regard de l'argile, qualité avantageuse dans des milieux humides.

Les récipients de stockage et les bols ont été très communément en usage dans toutes les maisons de la région jusqu'à il y a 20 ou 30 ans; depuis, ils ont peu à peu été 
remplacés par des récipients en zinc d'abord, en plastique ensuite. Notre recherche s'est effectuée à partir de l'observation d'exemplaires encore présents dans quelques maisons de la région, le plus souvent à l'état d'abandon. De plus, une enquête a été entreprise auprès de vieilles femmes qui les ont fabriqués et employés dans le passé. Quelques-unes d'entre elles ont accepté de reproduire ces récipients selon différents procédés de fabrication.

La fabrication

10 La fabrication de ces objets était exclusivement féminine. Cette région est aussi connue pour ses poteries modelées par des femmes (Vossen 1990; González et al., sous presse). Néanmoins, alors que cette poterie cuite relève de spécialistes, l'élaboration de récipients en bouse de vache et argile non cuite était une production domestique pratiquée par toutes les femmes de la région.

Dans le douar de Harrakah, on fabriquait des récipients de stockage cy-lindriques et des bols; les premiers avaient environ $40 \mathrm{~cm}$ de diamètre et de $1 \mathrm{~m}$ à $1,5 \mathrm{~m}$ de haut (photo 1), les seconds avaient de 30 à $40 \mathrm{~cm}$ de dia-mètre et $20 \mathrm{~cm}$ de profondeur (photo 2). Nous avons demandé à une femme du douar de reproduire le processus de fabrication des deux types de réci-pients, avec chacun ses spécificités. L'artisane avec qui nous avons travaillé était âgée d'environ 60 ans et pratiquait encore cette technique il y a une vingtaine d'années. La reconstitution s'est effectuée à côté de la porte d'entrée du patio, près de l'aire de stockage du bois et du foyer extérieur (photo 3). Autrefois, ce travail était réalisé dans n'importe quelle partie de la maison, à l'intérieur comme à l'extérieur. Notre informatrice, assise sur le sol, a utilisé des morceaux carrés d'écorce de chêne-liège comme support. La matière première était de la bouse de vache fraîche, prélevée directement dans l'écurie, sans mélange de paille. Autrefois, la bouse était stockée dans un puit d'un mètre de profondeur, situé hors de la maison.

Le bol est monté à l'aide d'un moule en céramique. Premier temps : la bouse est pétrie sur un sol poussiéreux. D'après l'artisane, la poussière argileuse donne de la solidité à la pâte; le fond de la céramique est également recouvert de poussière pour faciliter l'extraction du récipient en fin de montage. La pâte s'adapte à la forme du moule par une pression des poings contre le fond et les parois. Ensuite, les parois du bol sont montées avec des colombins de bouse posés contre les bords de la céramique. Cette technique produit une forme carénée caractéristique (photo 4). Puis la bouse est laissée à sécher au soleil dans le moule pendant 4 jours, avant d'en être extraite. Pour la finition, le bol, déjà solide, est enduit d'une couche d'argile humide et après séchage, d'une autre d'argile blanche (kaolin). Les deux couches sont étalées sur la surface du récipient directement à la main. La terre qui sert à engober les récipients est tamisée jusqu'à l'obtention d'une poudre fine; mélangée à de l'eau, elle est ensuite pétrie et étalée à l'intérieur et à l'extérieur de l'objet'².

Le récipient de stockage est monté au colombin. Les caractéristiques de la matière première, d'une moindre capacité de cohésion que l'argile, déterminent la technique employée. La femme prépare d'abord la base de l'objet sous forme de galette, sur une planche d'écorce de chêne-liège. Ensuite, les parois sont montées, non pas à l'aide de rouleaux de bouse continus, mais avec de petits colombins longs d'environ $10 \mathrm{~cm}$. Les colombins sont confectionnés en pétrissant des mottes de matière entre les mains, de manière à obtenir une forme fuselée. Le colombin est ensuite légèrement aplati d'un côté avec le tranchant de la main, afin que ce côté, légèrement concave, serve de base au positionnement de la matière sur les parois du récipient en cours de montage (photo 
5). La faible cohésion de la matière rend impossible le montage de façon continue; aussi, chaque jour, n'y sont consacrées que quelques minutes de travail qui permettent de monter 15 à $20 \mathrm{~cm}$ de paroi. L'objet est ensuite mis à sécher au soleil jusqu'au lendemain, puis l'opération est répétée.

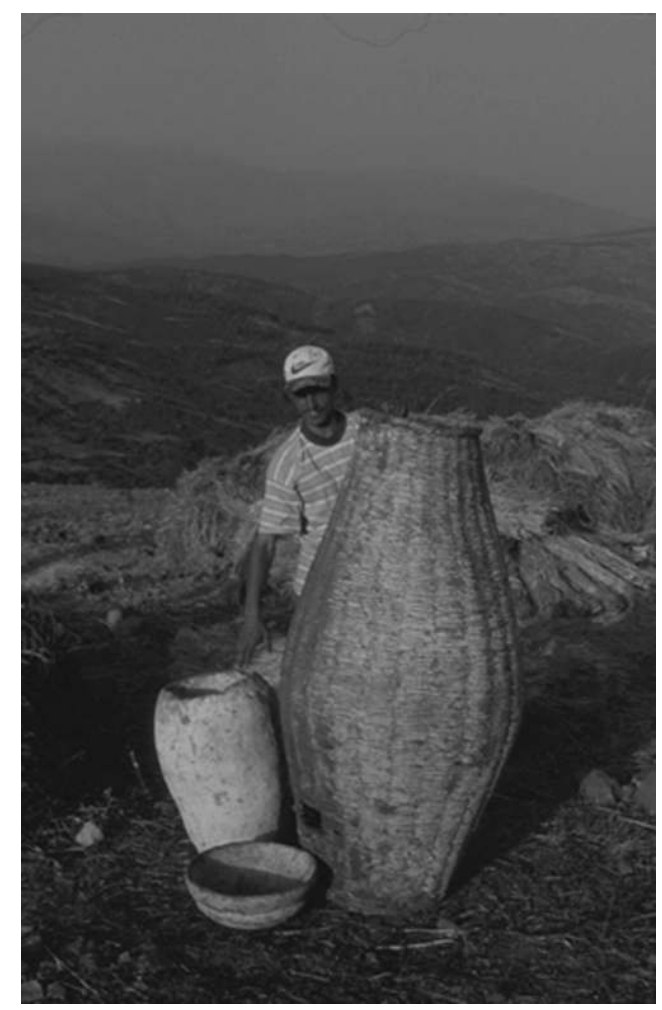

Photo 1. Récipients en bouse de vache, type cylindrique et bol (à gauche), et vannerie couverte par la bouse (à droite), à Harrakah

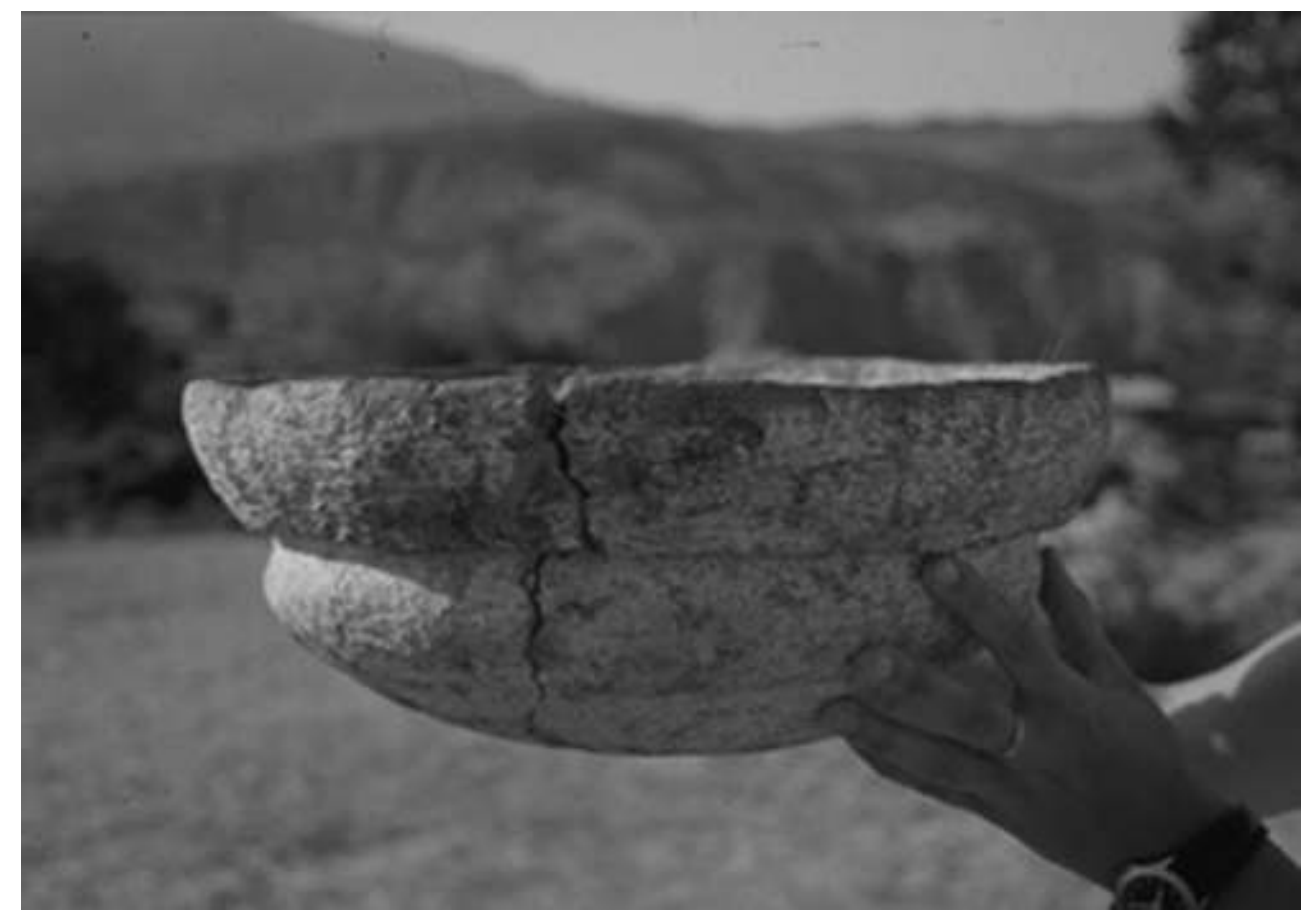


Photo 2. Récipient en bouse de vache employé pour donner à manger aux animaux et transporter le blé au moulin rotatif (douar Harrakah)

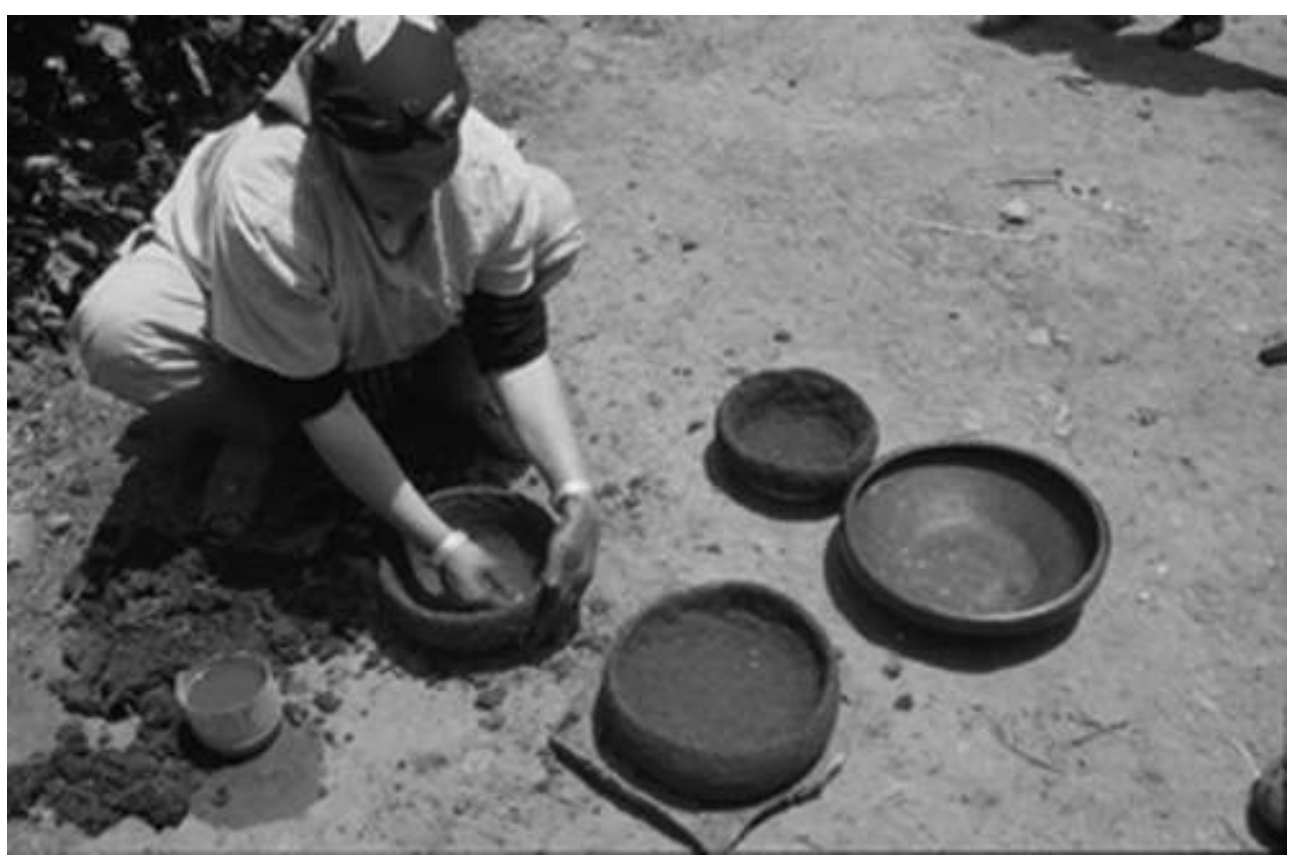

Photo 3. Fabrication des récipients à l'extérieur de la maison (douar Harrakah)

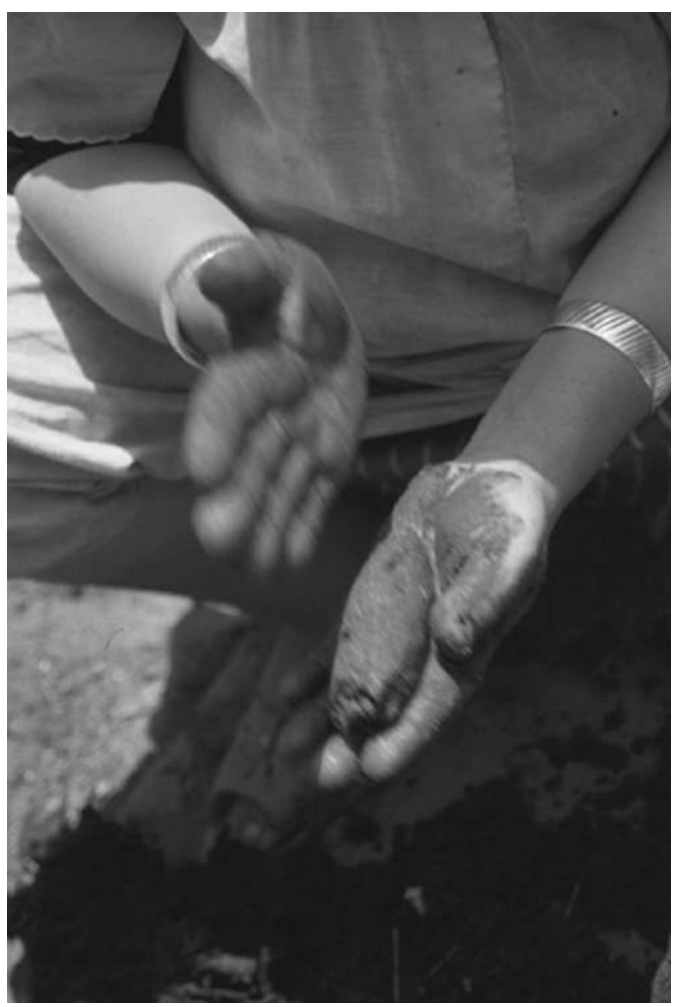


Photo 4. Préparation des colombins de forme fuselée pour le façonnage des grands récipients de stockage (douar Harrakah)

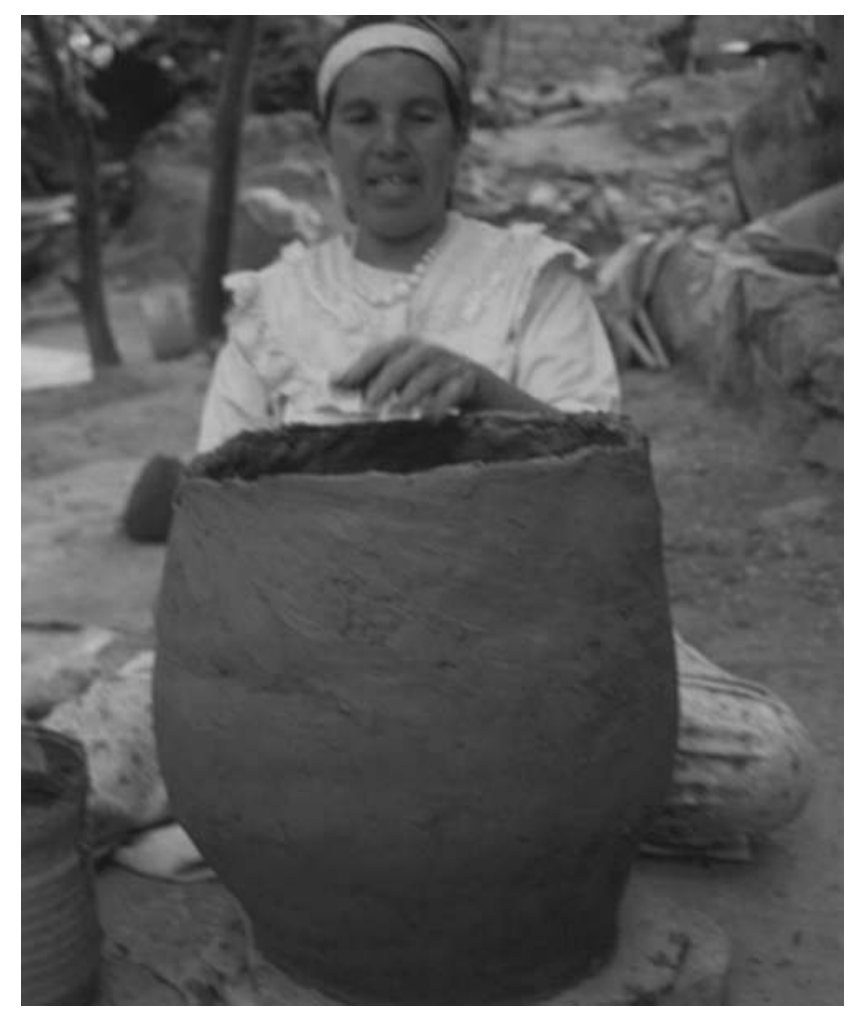

Photo 5. Élaboration d'un récipient cylindrique en bouse de vache mélangée avec l'argile à Ain Kob, selon la technique de Dar Hok

Le travail principal de façonnage est achevé en douze jours. Reste la finition, qui consiste à couvrir les parois internes et externes du récipient avec une première couche d'argile rouge puis une deuxième couche d'argile blanche. Tout le processus de fabrication dure 15 jours.

Dans le douar de Ain Kob, nous avons observé un processus de fabrication de récipients cylindriques faits d'un mélange d'argile et de bouse de vache réalisé par Mme Mnana, également experte en fabrication de poterie cuite. Cette artisane est originaire du douar de Dar Hok à $20 \mathrm{~km}$, situé à l'est d'Ain Kob où elle est venue s'installée il y a trente ans pour suivre son mari. A Dar Hok, où il y a des céramistes, elle a appris la technique de fabrication de la poterie cuite avec sa belle-mère et la pratique encore aujourd'hui.

À Dar Hok, on fabriquait et employait des récipients cylindriques de stockage des grains qui pouvaient mesurer de $1 \mathrm{~m}$ à plus de $2 \mathrm{~m}$ de haut, en bouse de vache seule ou mélangée à de l'argile. Le choix de l'un ou l'autre des matériaux dépendait de plusieurs paramètres. Au printemps, les animaux se nourrissent d'herbe fraîche; la bouse est alors souvent trop liquide: on doit donc y ajouter de l'argile. La bouse était aussi mélangée à de l'argile lorsque la quantité de matière animale était insuffisante. Les conditions climatiques avaient aussi un rôle déterminant: en effet, des températures élevées sont nécessaires au séchage des récipients en bouse de vache. C'est pourquoi on utilisait la bouse seule pendant la saison chaude et on la mélangeait lorsque le temps était plus humide et frais. 
17 Tous les récipients étaient fabriqués suivant les mêmes procédés et avaient la même fonction. Simplement, ceux en bouse et argile étaient plus lourds. Mais il faut noter que les récipients cylindriques en bouse de vache sont considérés comme spécialement appropriés au stockage des céréales, car ils les conservent à une température stable. De plus, ils sont très légers et, sauf cassure accidentelle, durent fort longtemps. Un de ces objets, que nous avons vu à Harrakah, avait plus de 40 ans. Toutefois, en bouse ou en argile, ils ne peuvent ni contenir des liquides ni entrer en contact avec le feu. D'où le fait que les poteries ont toujours été indispensables, et fabriquées et utilisées parallèlement aux tonna et tabtoba.

Nous avons demandé à Mme Mnana de reproduire la technique de fabrication des récipients en argile avec bouse (photo 6), qu'elle pratiquait avant de se marier, alors qu'elle habitait Dar Hok. Semblable à celle que nous avons déjà décrite, la technique mise en œuvre présente néanmoins quelques différences de détail. Ici, les parois ne sont pas façonnées à partir de petites mottes, mais avec des colombins continus et cylindriques montés à la main; elles sont ensuite affinées et régularisées avec des spatules en bois. Les récipients peuvent, ou non, être recouverts d'argile blanche.

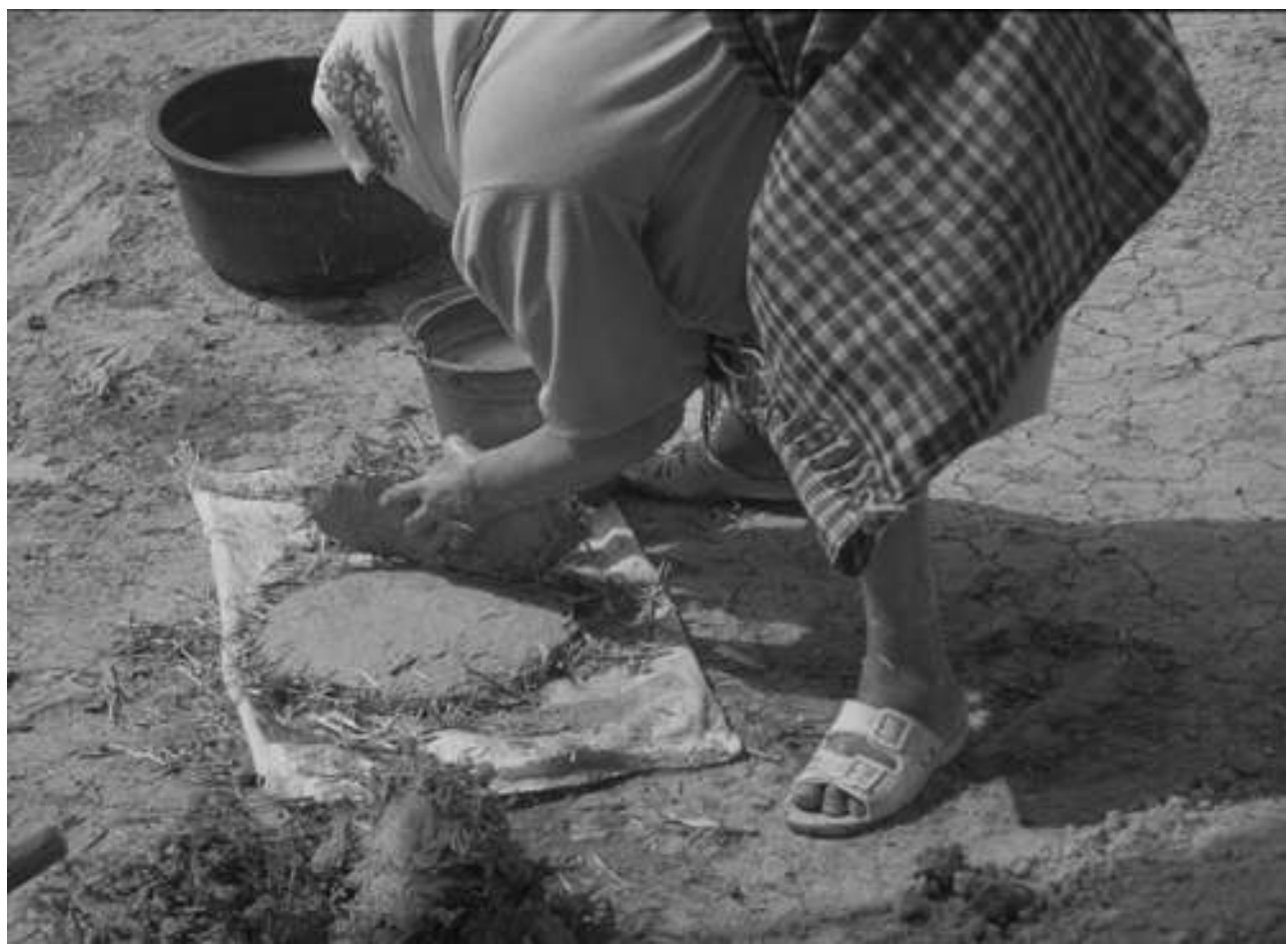


Photo 6. Élaboration d'un récipient cylindrique en argile à Kidiua Hainuna. Préparation de la basse du récipient

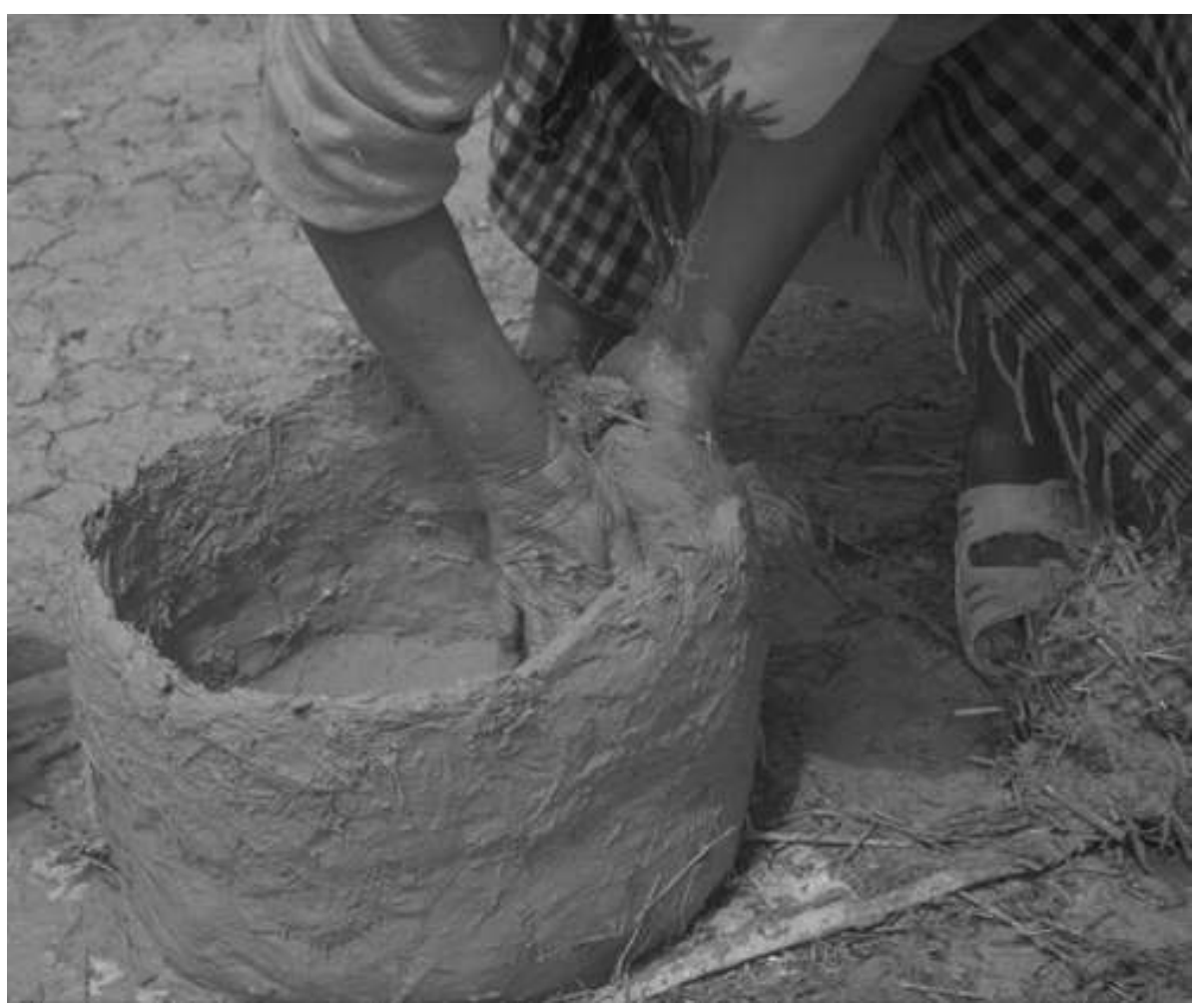

Photo 7. Élaboration d'un récipient cylindrique en argile à Kidiua Hainuna. Montage des parois du récipient 
délimiter une ouverture de $20 \mathrm{~cm}$ de diamètre. Cette ouverture est fermée par un couvercle. Sur la partie haute du récipient, se trouvent deux anses diamétralement opposées. Le tonna est recouvert d'une couche d'argile blanche dont la finalité est esthétique. Cette argile est ramassée au douar de Boukia, à 30 minutes à pied, là où les céramistes de Ksera, village distant de $12 \mathrm{~km}$ de Kidiua Hainuna, s'approvisionnent en argile pour décorer les poteries.

Autrefois, les récipients de plus grande taille ( $2 \mathrm{~m}$ de haut ou plus) étaient fabriqués dans la pièce où ils allaient rester fixés au sol par de l'argile. Les plus petits, qui pouvaient être déplacés, étaient montés soit dans le patio de la maison, soit à l'extérieur; ils étaient posés sur une couche de paille qui les isolait du sol.

Outre la paille, d'autres matières pouvaient donner consistance aux récipients cylindriques d'argile. À Oued Laou par exemple, les récipients de stockage étaient fabriqués en argile mélangée à de petits galets, les mêmes que ceux employés pour la construction des fours à poterie. Les parois étant assez épaisses $(4 \mathrm{~cm})$, les récipients ainsi fabriqués étaient très lourds. Leur fa-çonnage se déroulait à l'extérieur de la maison; puis, une fois secs, à l'aide des filets habituellement utilisés pour transporter la paille et l'herbe, ils étaient amenés à l'intérieur de la pièce de stockage.

Il existait aussi des récipients fabriqués exclusivement en argile, comme dans les douar de Slit et Dar Hok. Dans ce cas, le séchage du récipient était réalisé à l'ombre et durait environ un mois, afin d'éviter le fendillement des parois. Pour tous les types de récipients en argile et bouse de vache, l'été constituait la période de travail privilégiée par les femmes car la chaleur, nous l'avons dit, est une composante importante du processus.

La fonction

Les récipients cylindriques étaient destinés au stockage de produits alimentaires secs : céréales, légumineuses, farine, raisins secs, figues sèches, sel, etc. Leur taille était fonction des produits à conserver. A Oued Lau, par exemple, les plus petits - contenant 30 à 40 kilos- étaient utilisés pour garder les fèves, les moyens -autour de 100 kilospour les figues et les céréales, les plus grands -jusqu'à 300 kilos- pour les grains uniquement.

Les récipient étaient fermés par une planche en bois ou en écorce de chêne-liège; ils pouvaient aussi être scellés avec de l'argile. Pour les plus grands - plus de 1,5 $\mathrm{m}$ de haut - , on y pratiquait une ouverture, avec un couteau, près de la base, pour en faire sortir les denrées. Le trou était ensuite rebouché à l'aide de chiffons, parfois avec de l'argile.

Ces récipients étaient entreposés dans la pièce de stockage, qui pouvait par ailleurs assumer d'autres fonctions. Ceux en argile -les plus grands et les plus lourdsrestaient fixés au sol; pour les isoler de l'humidité, ils étaient posés sur une couche de paille ou étaient surélevés sur des pieds en argile ou en pierre et argile. À Harrakah une famille pouvait posséder six ou sept récipients, un pour chaque type de produit, tandis qu'à Kidiua Hainuna, chaque maison en comptait jusqu'à quinze. D'une manière générale, les besoins en récipients étaient plus importants les années de bonnes récoltes.

Différents facteurs expliquent l'emploi de ces récipients cylindriques. D'une part, la fabrication des grandes poteries nécessaires au stockage se heurtait aux limites techniques du savoir-faire des femmes céramistes de la région : d'après elles, il est impossible de fabriquer de très grandes poteries pour stocker les produits de la récolte. 
D'autre, part, on stockait également les denrées, toujours à l'intérieur des maisons, dans de grands récipients ovales en vannerie, pouvant atteindre $2 \mathrm{~m}$ de haut (Lefébure 1979). Ceux-ci étaient recouverts d'argile blanche ou de bouse pour assurer la conservation du produit et éviter que les grains ne tombent par les interstices de la vannerie. Nos informatrices reconnaissent que les récipients en vannerie avaient de plus grandes capacités de stockage. Mais la vannerie est un travail de spécialiste masculin et doit donc être achetée au souk, sauf si un homme de la famille en maitrise la technique.

Dans les zones à sol d'argile imperméable, les grains étaient également stockés dans les matmora, silos souterrains aux parois tapissées de paille. Ces silos servaient en cas de récolte très abondante, puisqu'ils permettaient de stocker plusieurs tonnes de grain. Mais ils constituaient des structures de stockage à usage différé, car ils ne pouvaient être ouverts de façon répétée et continue, sous peine de détérioration du produit stocké. Les récipients cylindriques étaient destinés à des quantités plus réduites, mais on y avait accès de façon continue. C'est pourquoi, dans certains douar, on les utilisait conjointement aux matmora.

Les bols étudiés dans les douar de la région de Tanakoub avaient deux fonctions :

- nourrir les animaux (vaches, mules et chèvres) dans l'écurie; là, il y a des mangeoires carrées ou semi-circulaires formées de murs bas, où le récipient empli de nourriture était déposé; quand l'animal avait fini de manger, le récipient était rangé dans un coin de l'écurie;

- transporter les grains à moudre du lieu de stockage jusqu'au moulin rotatif manuel; le bol était rangé à côté du moulin situé dans une des pièces du rez-de-chaussée de la maison.

Dans chaque maison, on disposait de quelques récipients de ce type, trois ou quatre pour la mouture des céréales et un pour chaque animal de l'écurie. Aujourd'hui, les récipients en bouse sont remplacés par d'autres, en vannerie ou en plastique.

Les contenants cassés pouvaient être réemployés pour le ramassage du miel. Les morceaux étaient introduits à l'intérieur de céramiques en forme de bouteille, destinées à cette collecte. On mettait le feu aux fragments de bouse et l'on soufflait par un trou situé à la base de la bouteille, de sorte que la fumée s'échappât par la bouche de la céramique, éloignant ainsi les abeilles.

Parallèles ethnographiques

L'emploi de différents types de récipients pour conserver les produits agricoles (céréales, légumineuses, fruits...) à l'intérieur des maisons est largement connu dans les sociétés traditionnelles des régions méditerranéennes. Ces récipients sont habituellement fabriqués en peau, tissu de laine, céramique, sparterie, vannerie... (Ferchiou 1979; Louis 1979; Lefébure 1979; Ayoub 1979). En revanche, l'utilisation de l'argile non cuite et de la bouse est plus rarement citée.

Pour les Jbâla, nous n'avons trouvé que deux références. Dans Mots et choses berbères, d'Emile Laoust (1920:68), au chapitre sur la céramique de la région, l'auteur note en pied de page : «On nous signale que les Aït Warain utilisent des poteries non cuites et simplement séchées au soleil. Le fait aurait cependant besoin d'être vérifié sur place ». Al-Ayachi Al-Mrini (1984), dans son travail sur les principales spécialités artisanales des tribus Jbâla, Ghomara et Sanhaja, indique que la tribu Ghzawa (région de Mokrisset) fabrique une poterie séchée, non cuite.

36 La bouse de vache est pourtant largement employée dans les communautés paysannes (Makal 1954; Reddy 1994; Ertug-Yaras 1997; D’Andrea et al., sous presse; Butler et al., 
sous presse). Dans la région de Chefchaouen, on en couvre l'aire de battage en été et, mélangée à de l'argile, on s'en sert pour enduire les sols et les murs des maisons. Sèche et sous forme de galette, elle sert à couvrir les fours de cuisson ouverts des potières et comme support de montage des poteries, généralement en mélange avec de l'argile. Des emplois similaires ont été reconnus, par exemple à Tigrayan, dans le nord de l'Éthiopie (A. Butler, com. pers.).

Il existe de nombreuses références concernant l'usage de la bouse en relation avec le stockage de la nourriture. Au Maghreb, elle est utilisée pour enduire les silos souterrains, car on lui attribue des propriétés de protection contre les insectes (de Luca 1979). Dans la région de Konya (Turquie), de grandes réserves à structure en coupole sont construites avec des galettes sèches de bouse couverte d'un mélange d'argile et de bouse (J. Juan, com. pers.). Dans les régions montagneuses du nord de l'Éthiopie, on trouve des structures de stockage construites avec de la bouse et de l'argile, qui mesurent de $1 \mathrm{~m}$ à $1,5 \mathrm{~m}$ de haut et sont adossées aux maisons (A. Butler, com. pers.). Dans la région de Chefchaouen, les grands récipients en vannerie pour le stockage des grains sont couverts de bouse, procédé également connu dans quelques régions du sudest de l'Asie (J. Thompson, com. pers.) et en Inde (Reddy 1994, s/presse).

Les excréments de différents animaux (cheval, vache, lapin et âne) peuvent être mélangés à de l'argile dans le processus d'élaboration des poteries (London 1981; Gelbert 1999). En revanche, nous avons trouvé très peu de références concernant la bouse pour le montage de récipients non cuits. Il existe des notes dans les textes des agronomes arabes médiévaux Abu Zacharia et Abu l-Jayr. Ils recommandent cette matière pour conserver les raisins secs, en affirmant que ce système en permettait la conservation jusqu'au début du mois de juin. Ces procédés ont été évoqués d'une façon très sommaire par Makal (1954) qui les a repérés dans un petit village d'Anatolie. G. Hillman (1984) signale l'existence, dans cette même région, de grands récipients en bouse de vache pour contenir le bourgour. Récemment, dans le petit village turc de Kizilkaya, Ertug-Yaras (1997) a trouvé quelques récipients en bouse, abandonnés dans les étables et les zones de stockage; il s'agit d'objets de 15 à $20 \mathrm{~cm}$ de diamètre et de 12 à $20 \mathrm{~cm}$ de haut. Quelques vieilles femmes l'ont informé que ces récipients étaient destinés à contenir des œufs ou des cendres balayées sur le sol. Les générations les plus jeunes n'en fabriquent et n'en utilisent plus.

39 Pour ce qui est des objets en argile, dans le petit village iranien de Aliabad (Kramer 1982), des récipients ronds et rectangulaires en argile ou en torchis ont été signalés. Utilisés également pour stocker des produits agricoles, ils sont gardés dans la cuisine et présentent un trou à leur base qui permet d'en extraire le contenu. Ils restent fixés au sol et sont isolés de celui-ci par des pieds en pierre ou en argile. À l'exception des récipients mobiles, qui font ici défaut, ces caractéristiques sont proches de certains exemplaires jebli de la région Jbâla.

Un processus technique de type domestique

Résumons-nous. Le principal avantage des récipients en bouse de vache et en argile non cuite est leur caractère multifonctionnel au regard de la simplicité du processus de fabrication. Les bols en bouse de vache, légers et résistants, sont d'un usage quotidien. Les récipients cylindriques servent à stocker les produits agricoles dans la maison. $\mathrm{Si}$ les silos souterrains (matmora) sont plus aptes au stockage d'importantes quantités de grain à plus long terme, les récipients en argile et bouse permettent un accès continu à 
des quantités plus réduites, et donc une souplesse de la redistribution adaptée aux besoins quotidiens.

41 D'autres modes de stockage sont connues, comme les grandes vanneries, fabriquées par des spécialistes; il s'agit alors d'une production qui doit être achetée. Dans une économie plutôt autarcique, comme l'était celle la montagne jebli il y a quelques décennies, on préférait une production domestique des récipients. La simplicité technique de la production en argile ou en bouse autorisait ce choix. D'une part, la matière première est facilement accessible pour n'importe quelle communauté paysanne gérant un troupeau de bovins. D'autre part, le processus de travail n'exigeait pas de connaissances techniques sophistiquées, qui auraient imposé un long apprentissage et des structures aménagées. Ce savoir était, de fait, partagé par toutes les femmes de la région qui confectionnaient ces récipients pour leur propre consommation. La transmission du savoir-faire se faisait donc au sein de la maison, au même titre que n'importe quelle activité domestique féminine.

Visibilité archéologique

S'agissant d'une technique simple, il est probable que le processus de fabrication décrit ici ait existé parmi des communautés de paysans préhistoriques. Mais la visibilité archéologique de ces objets est nécessairement fort réduite, puisque la bouse est une matière organique périssable, et l'argile non cuite une matière très fragile.

Des récipients en argile non cuite ont cependant été repérés dans quelques sites du Néolithique ancien du Proche-Orient. Les exemples les plus anciens correspondent aux sites PPNA de Tell Mureybet, en Syrie (Cauvin 1974) et de Demirköy, en Turquie (Rosenberg \& Peasnall 1999). Sur le site PPNB de Çayönü, différents types de contenants en argile non cuite mélangée avec la paille ont été mis au jour. Quelques-uns sont peu profonds, à base plane, et de morphologie rectangulaire ou ronde. D'autres sont plus profonds et présentent des traces de vannerie de roseaux à l'extérieur (Ózdogan \& Ózdogan 1993; Ózdogan, A. 1999). Dans les niveaux du PPNB moyen de Tell Halula (Molist 1996), on a découvert quelques récipients cylindriques en argile, dans une des cellules interprétées comme chambres de stockage des maisons rectangulaires. Des récipients en argile et paille ont été également trouvés dans quelques sites datant de la première moitié du 7ème millénaire avant J.-C. en Mésopotamie (Wengrow 1998 : 786). Des récipients de stockage en argile ont été repérés à l'intérieur des maisons sur le site Chalcolithique de Podgorista (Bailey et al. 1998 : 390).

Les possibilités de préservation dans les sites archéologiques des récipients en bouse devraient être les mêmes que celles des autres matières organiques : par dessiccation, par immersion et par carbonisation. Les deux premières conditions sont exceptionnelles; c'est pourquoi nous nous sommes attachés aux possibilités de préservation de ces objets par carbonisation.

Afin de tester les possibilités de conservation, nous avons développé une expérimentation ciblée. Plusieurs récipients, fabriqués en bouse de vache, entourés d'une fine couche d'argile et en bouse mélangée avec de l'argile, ont été jetés à différents endroits d'un petit foyer. Les premiers ont été détruit lors de la combustion. En revanche, les seconds, lorsqu'ils étaient proches du feu, ont été céramisés par la chaleur. Ils ont alors été abandonnés en plein air. Au bout d'un an, même si leur pâte cuite était assez grossière, les tessons correspondants sont restés conservés. De ces premières expériences, on peut conclure à une conservation archéologique différentielle par carbonisation entre les objets réalisés en bouse, qui disparaissent, et 
ceux réalisés en argile mélangée avec la bouse, qui, dans certaines conditions de chaleur conduisant à une "céramisation» de leur pâte, ont des chances de se conserver.

Somme toute, la simplicité des techniques décrites laisse supposer qu'elles ont pu être largement employées aux temps préhistoriques, mais il ne s'agit encore que d'une hypothèse. La conservation problématique des récipients ainsi obtenus rend difficile leur identification. Il nous faut cependant toujours garder cette image à l'esprit, sinon comment les rechercher?

Nous tenons à remercier J. Vignet-Zunz, J. Juan, A. Butler et J. Thompson pour nous avoir fourni nombre d'informations, et $\mathrm{S}$. Beyries pour ses commentaires sur une version préliminaire de cet article; $\mathrm{H}$. Essouifi pour son dévouement et son intelligence durant le travail sur le terrain ainsi que $\mathrm{C}$. Llaty et $\mathrm{S}$. Beyries pour avoir corrigé la version en français. À tous, notre amitié.

\section{BIBLIOGRAPHIE}

\section{Al-Ayachi AL-Mrini}

1984 Suwarun min al-tarikh al butuli li mintaqat Jbala. Tanger : Imprimerie Marocaine et Internationale,

Ayoub, Abderrahman

1979 « Les moyens de conservation des produits agricoles dans le nord-ouest de la Jordanie actuelle », pp. 155-169, in M. Gast et F. Sigaut (eds), Les techniques de conservation des grains à long terme. Paris : Éditions du CNRS.

Bailey, DouglasW. \& Ruth Tringham et al. 1998 « Expanding the dimensions of early agricultural tells: the Podgoritsa archaeological project, Bulgaria », Journal of Field Archaeology 25 (4) : 373-396.

Cauvin, Jacques

1974 « Les débuts de la céramique dans le Moyen Euphrate : nouveaux documents ", Paléorient 2 : 199-203.

Chikhi, Nourredine E. \& Mohamed el Abdellaoui 1996 « El regadío y las transformaciones agrarias en el Rif », pp. 255-282, in J. A. González Alcantud, M. González de Molina, A. Malpica, et J. Vignet-Zunz (eds), Transformaciones agrarias y cultura material en Andalucía oriental y norte de Marruecos. Diputación Provincial de Granada y Ministerio de Agricultura.

Ertug-Yaras, Fusun

1997 An Ethnoarchaeological Study of Subsistence and Plant Gathering in Central Anatolia. Ph. D. Thesis. Washington University.

Ferchiou, Sophie

1979 « Conserves céréalières et rôle de la femme dans l'économie familiale en Tunisie », pp. 190-197, in M. Gast et F. Sigaut (eds), Les techniques de conservation des grains à long terme. Paris : Éditions du CNRS. 
Gelbert, Agnès

1999 « Technological and Stylistical Borrowing Between Ceramic Traditions : A Case Study from Northeastern Senegal », pp. 207-224, in L.R. Owen y M. Porr (eds), Ethno-Analogy and the Reconstruction of Prehistoric Artefact Use and production. Tübingen : Urgeschichtliche Materialhefte, 14.

Gonzáles Urquijo, Jesús, Juan José Ibáñez, Lydia Zapata \& Leonor Peña-Chocarro 2001 « Estudio etnoarqueológico sobre la fabricación de la cerámica en la región de Mokrisset (Chefchauen, Marruecos). Aspectos técnicos y sociales de un artesanado arcaico ", Trabajos de Prehistoria 58 (1) : 5-29.

Kramer, Carole 1982 Village Ethnoarchaeology. Rural Iran in Archaeological Perspective. London/New York : Academic Press.

Laoust, Emile

1920 Mots et Choses Berbères. Paris : Agustin Challamel.

London, Gloria

1981 « Dung-tempered clay », Journal of Field Archaeology 8 : 189-195.

Louis, André

1979 « La conservation à long terme des grains chez les nomades et semi-sédentaires du Sud de la Tunisie ", pp. 205-214, in M. Gast et F. Sigaut (eds), Les techniques de conservation des grains à long terme. Paris : Éditions du CNRS.

Luca (de), Yvon

1979 « Ingrédients naturels employés dans les silos locaux des pays en développement », pp.

147-150, in M. Gast et F. Sigaut (eds), Les techniques de conservation des grains à long terme. Paris : Éditions du CNRS.

Makal, Mahmut

1954 A Village in Anatolia. London: Mitchell and Co (Translated by Wyndham Deedes, Vallentine).

Molist Montaña, Miquel (ed.)

1996 Tell Halula (Siria). Un yacimiento neolítico del valle medio del Eufrates. Campañas de 1991 y 1992. Madrid : Ministerio de Educación y Cultura.

Ózdogan, Mehmet \& Asli Ózdogan

1993 «Pre-Halafian Pottery of Southeastern Anatolia with Special Reference to the Çayönü Sequence ", pp. 87-103, in Between the Rivers and Over the Mountains. A. Palmieri Dedicata. Rome.

Ózdogan, Asli

1999 « Çayönü », in M. Özdogan et N. Basgelen (eds), Neolithic in Turkey. The cradle of civilization. New discoveries. Istanbul : Arkeoloji ve Sanat Yav.

Reddy, Seetha.

1994 Plant Usage and Subsistence Modeling : An Ethnoarchaeological Approach to the Late Harappan of Northwest India. Doctoral Dissertation University Microfilms, Ann Arbor.

s/presse Discerning Palates of the Past: An Ethnoarchaeological Study of Crop Cultivation and Plant Usage in India. International Monographs in Prehistory. Ann Arbor : Ethnoarchaeological Series.

Rosenberg, Michael \& Brian Peasnall

1999 « A Report on Sounding at Demirkoy Höyuk : an Aceramic Neolithic Site in Eastern Anatolia », Anatolica, XXIV : 195-207. 
Vignet-Zunz, Jacques

1993 « Manifestations insolites dans le patrimoine technique des Jbâla (Maroc) », Jornadas

Internacionales sobre Tecnología Agraria Tradicional. Madrid : Museo Nacional del Pueblo Español.

Vossen, Rüdiger

1990 Reisen zu Marokkos Töpfern. Hamburg.

Wengrow, David

1998 « The changing face of clay : continuity and change in the transition from village to urban

life in the Near East », Antiquity $72: 783-795$.

\section{ANNEXES}

Figures

Figure 9: Récipient cylindrique en argile elaboré à Kidiua Hainuna.

Figure 10: Utilisation des récipients pour la nourriture du bétail, douar Harraka.

Figure 11: Récipient fuselé en argile employé pour stocker les produits agricoles à Ifran Ali (Oued Lau).

\section{NOTES}

1. Ce projet, intitulé L'apport de l'ethno-archéologie au Maroc pour la connaissance des premières sociétés productrices, est financé par la Fundación Marcelino Botín. À l'occasion de trois missions de recherche sur le terrain (1997, 1998 et 1999), nous avons travaillé sur la culture du Triticum monoccocum, la poterie montée au colombin par les femmes, les systèmes de tannage de la peau et la construction des maisons en torchis. 2. Les récipients les plus petits pouvaient se passer de l'engobage.

\section{RÉSUMÉS}

Dans la région Jbâla, au nord-ouest du Maroc, nous avons étudié les techniques traditionnelles de fabrication de récipients réalisés 1) en bouse de vache, 2) en argile non cuite, et 3) en argile mélangée avec la bouse de vache. La fabrication de ces objets, abandonnée depuis vingt ou trente ans, reposait sur le savoir-faire technique de toutes les femmes de la région et avait pour but de satisfaire les besoins domestiques. Autrefois, ces récipients servaient au stockage des produits agricoles secs, au nourrissage des animaux ou encore au transport des céréales au moulin rotatif.

Clay and cow dung. The earthenware containers of the Jbâla region (Morocco)

In the Jbâla region, North Western Morocco, we have studied the traditional making of containers (tonna and tabtoba) from 1) cow dung, 2) unfired clay, and 3) clay mixed with cow dung. The making of these objects for domestic use represented a general technical knowledge shared by all women in the region and disappeared twenty or thirty years ago. These containers 
were used for storing cereals and other dry products, for feeding livestock and for transporting cereal to the quern.

Arcilla y boñiga. Los recipientes de la región jbâla (Marruecos)

En la región jbâla, en el noroeste de Marruecos, hemos estudiado las técnicas tradicionales de fabricación de recipientes realizados 1) en boñiga, 2) en arcilla cruda y 3) en arcilla mezclada con boñiga. La producción de estos objetos, que se abandonó hace veinte o treinta años, se basaba en el "saber hacer" técnico de todas las mujeres de la region, y respondía a las necesidades domésticas. Antes, esos recipientes servían para almacenar productos agrícolas secos, tanto destinados a la alimentación humana como animal, o también para transportar en ellos los cereales al molino rotativo.

\section{INDEX}

Mots-clés : argile crue, bouse de vache, Jbâla, Maroc, poterie

\section{AUTEURS}

\section{JUAN JOSÉ IBÁÑEZ}

Departamento de Ciencias Históricas. Universidad de Cantabria. Avda. de los Castros, s.n., Santander. ibanezjj@unican.es

\section{LEONOR PEÑA-CHOCARRO}

Laboratorio di Archeobiologia. Musei Civici di Como.Piazza delle Medaglie d'Oro 1.22100 Como, Italy. leonorpc@libero.it

\section{LYDIA ZAPATA}

Department of Archaeology. University of Cambridge. Downing Street. CB2 3DZ. lz208@hermes.cam.ac.uk

\section{JESÚS EMILIO GONZÁLEZ URQUIJO}

Departamento. de Ciencias Históricas. Universidad de Cantabria. Avda. de los Castros, s.n., Santander.gonzalje@unican.es

\section{MARTA MORENO GARCÍA}

Instituto Portugués de Arqueología. Avda. da Índia 136. 1300-300 Lisboa, Portugal. marta@ipa.min-cultura.pt 